\title{
An Emerging Digital Ecosystem: Blockchain Competence Certification Networks
}

\author{
Roberta Cuel ${ }^{1}$, Francesco Virili ${ }^{2}$, Cristiano Ghiringhelli ${ }^{3}$, Francesco Bolici ${ }^{4}$ \\ ${ }^{1}$ University of Trento, Via Inama 5, 38123 Trento, Italy \\ ${ }^{2}$ University of Sassari, Piazza Università 21, 07100 Sassari, Italy \\ ${ }^{3}$ University of Milano-Bicocca, Piazza dell'Ateneo Nuovo 1, 20126 Milan, Italy \\ ${ }^{4}$ University of Cassino and Southern Lazio, Loc. Folcara, 03043 Cassino, Italy \\ roberta.cuel@unitn.it, fvirili@uniss.it, \\ cristiano.ghiringhelli@unimib.it, f.bolici@unicas.it
}

\begin{abstract}
In this paper, we investigate how blockchain technologies improve the certification system and generate added value for different involved actors: learners, educational institutions, and businesses. An exploratory study is proposed to systematize the overall impacts of blockchain in the field of digital certification while focusing on university education as the main research field. We carried out a desk analysis, a direct observation/focus groups and semi-structured interviews with key players of the two Italian universities that first adopted a blockchain-certification system. The aim is to make sense of drivers and value generation conditions in the new scenario introduced by the development of DACS (Digital Academic Certification System), shedding light on the relationships between actors in the ecosystem that characterize the different types of complementarities between actors and services before and after the introduction of blockchain-based platforms and the personal wallet containing titles and certifications. Three main findings arose from the study, related to actors, ecosystem and complementarities. The main contributions of this study from the theoretical point of view is that it produces a first empirical evidence to the new Ecosystem Theory proposed by Jacobides et al. [1]. From the managerial perspective, this study contributes to better identify and debate conditions and success factors unlocking value generation and benefits embedded in Digital Certification Ecosystems.
\end{abstract}

Keywords: Digital ecosystems, Blockchain, Competence Certification

\section{Introduction}

The need for specific skills and competencies evolves in response to environmental, social and organizational conditions. However, the model for certifying competence levels has remained almost unchanged for centuries: universities (and other institutions) verify and certify that a person has reached a certain level of knowledge and through a 
stand-alone solution. The output is often a signed and stamped document that undoubtedly contains limitations in the present international, multilingual, and dynamic job market. Studies on skills and competencies have often taken for granted that universities and other educational institutions would continue to act as independent certification bodies. This traditional certification routine is now under pressure. On the one hand, there are new actors (not necessarily universities or traditional educational institutions) in the education and training market. On the other, technological progress and globalization, to name just two factors, demand a radical innovation of the whole educational sector and thus introduce new competitive challenges for traditional institutions.

Universities are now at the centre of this challenging scenario where tensions exist among legacy systems, European and national regulations, learner empowerment and an increasing number of students who complete their learning programs across different institutions with different learning techniques.

Innovative forms of competence certification, enabled by digital platforms and in particular blockchain systems, are playing an increasingly relevant role. Here universities, students, and firms show diverse interconnections, value perceptions, and drivers

Blockchain has recently become studied across a broad range of sectors: from finance to healthcare, from tourism to public administration. A main appeal of blockchain is the possibility to conclude transactions without the need for a central authority, thus to operate along a decentralized model without increasing the risks embedded in the transaction. As synthesized by Bolici [2], its specific design (a mix of cryptography, governance model, distributed computer network and individual economic incentives) defines blockchain as an enabler for trustless transactions: every actor does not need to trust anyone else (the other part of the transaction or a central authority) to conclude $\mathrm{her} / \mathrm{his}$ arrangement. The absence of intermediaries and a strong anti-tempering system makes possible a transaction system without central authority.

In this paper, as first step of a structured research agenda, the theory of ecosystems recently proposed by Jacobides et al. [1] is adopted to make sense of value generation drivers and conditions in the scenario depicted above. We conducted a first set of interviews and a focus group with key players from the Italian universities that first adopted a blockchain certification system.

On this basis, we propose an exploratory study aimed at shedding light on the ecosystem's relationships characterising the different types of complementarities between actors and services before and after the introduction of blockchain-based platforms and personal wallet (Digital Academic Certification System) embedding titles and certifications.

\section{Theoretical framework}

Interorganisational systems and networks are recognized as the dominant form in the post-Fordist era [3]. This transition has already produced several effects both at the organizational level - large number of merger \& acquisitions, outsourcing, business networks, online marketplaces - and at the social level - new communication forms, the 
emergence of social network, the virtualization of relationships and work, the emerging communities of practice [4].

Among these effects is the emergence of digital business ecosystems, in which both social and technical factors are simultaneously taken into consideration. The term Digital Business Ecosystem (hereafter DBE) was initially referred to a "...collaborative environment made up of different entities that co-create value through information and communication technologies [5]" (see also [6]).

The early notion of DBE has been catching attention in the practitioners world for several years [7], while still requiring further theoretical development and commonly accepted definitions in the academy of sciences. The last few years have clearly registered an increasing attention on ecosystems; for example, the bibliometric analysis in Suominen et al. [8] shows a citation network of over 300 selected papers on this topic.

In recent years, significant steps towards a systematic analysis of this concept have been accomplished. Useful reference points are studies from different perspectives of value co-creation in ecosystems $[9 ; 10 ; 11 ; 12 ; 13 ; 14)$.

Using a grounded-theory-based literature review, Senyo et al. [6] evidenced relevant gaps for future research and a specific need for theorization. Early partial answers to the need for a systematic understanding are given by de Reuver et al. [15], with a convincing comparative analysis of digital platforms and digital ecosystems, and by Gupta et al. [16] who classified different types of ecosystems (business, innovation, and digital). A recent study [1] gave an important contribution towards a theory of ecosystems, drawing a crystal clear distinction between ecosystems and entities like hierarchies, markets, alliances, networks or other forms of business aggregation: an ecosystem is typically formed by a set of actors experiencing (different degrees of) complementarities in production and consumption. The analysis of production/consumption complementarities, in this view, is the elective tool of investigation to understand and characterize an ecosystem, with a particular attention to the enabling role of modularity.

We are embracing this perspective in the analysis of the digital competence certification ecosystem, observing how the different actors and their complementarities are orchestrating the emergence of the ecosystem itself.

\section{$3 \quad$ Methodology}

In this study we carried out a systematic exploration of the ongoing development process of the Digital Academic Credentials System in the field of university education. We collected data through a three-step process: desk analysis; direct observation/focus groups; semi-structured interviews.

The desk analysis represents the initial collection of secondary data needed to frame our research work. We extensively collected and analysed the literature regarding:

1. the most common stream of studies in organization and ICT (Information and Communication Technology) identifying three main perspectives (see Section 2);

2. past experiences aimed at developing an innovative framework of competence certification systems, regulations, and ICT solutions (with a specific focus on blockchain). 
Two of the authors get involved in some discussions, round tables and focus groups about the development of a distributed digital system for competence certification in their own institutions. Thus, we were able to collect a set of direct observations on the motivations, gateways, triggers, obstacles and potentialities at different stages of the process. We recognize the potential bias of collecting data through participatory research $[17 ; 18]$, and we smooth them out by integrating such data with semi-structured interviews with key actors.

Thus, we developed an interview protocol to facilitate and guide semi-structured open-ended interviews. We conducted a first set of interviews with key players that are promoting a blockchain-certification system. The interviews were focused on value creation factors and conditions for the different entities of the blockchain certification ecosystem. We recorded the interviews and all the researchers listened to them for later discussion. All the collected data were analyzed individually by each researcher, and then discussed and structured together. Significant episodes retold by the interviewer emerged and were then matched to organizational elements. In the following section we highlight some key episodes and point out their organizational relevance.

\section{$4 \quad$ Main and expected findings}

The main findings evidenced by analysis in progress are the following.

The first finding is a reconstruction of the actions taken by the first mover in the field. A sort of brief history in which actors playing an important role in the Italian ecosystem are described.

The second finding concerns ecosystems and its changes. It describes whether and to what extent the relationships among actors may change with the introduction and the adoption of Digital Academic Credential Systems (DACSs) based on blockchain. Consistent with this model, some dynamic isomorphic tensions are described underlying the way in which national and international institutions are introducing new norms to boost the adoption of DACS in Europe and USA.

The third finding considers the digital ecosystem complementarities, providing a more specific analysis of the relationships between actors in the ecosystem.

\subsection{A brief history}

In 2016, the University of Milan-Bicocca (hereafter UniMiB) started a pilot project to develop a Digital Academic Credential system. The aim was to build an innovative model enabling universities to digitally certify learners' competences, skills and participation activities. This service is based on an innovative concept that is spreading in Europe and in all the Western Countries: the digital academic credential system, in some cases called learner wallet or badge wallet.

Three key requirements lead the system development:

1. Self-verification: each certificate can be verified without the involvement of the issuer;

2. Incorruptibility: to ensure that the certificate is not counterfeit; 
3. Autonomy: the digital certificate, once verified, remains valid even if the issuer ceases to exist.

The interoperability among all the interested universities is a key outcome of this Digital Academic Credential System (DACS) to facilitate student exchange, ECTS recognition and authentication, especially at European level. During the interviews, one of the experts mentioned estimations of around 60 million students moving from emerging countries to advanced economies university systems in the near future. If confirmed, this trend will dramatically challenge the existing structure and organization of the university system. As a consequence, the role of digital learning is becoming more and more important, both in providing a partial solution to the high number of students as well as sustaining "stackable" degree programs between academic institutions and learning platforms. In this case the ownership of the credentials are of the learners that can combine all the certificates in one unique and verified system or wallet.

According to the process depicted in Figure 1, the increasing effort on soft skill development recommended by several European institutions, including the European University Association, strongly triggered the project aimed at developing a Digital Academic Credential system at UniMiB. Ideally the DACS enables learners to collect certificates from various educational issuers, organizes all the certification in one unique system and enables to share to third parties the certification and the related competences acquired.

In the following section we will explain better how the ecosystem will change with the advent and the massive adoption of the DACSs.

As depicted in Fig. 1, a couple of years ago UniMiB introduced the focus on soft skills development as a key issue in its 3-year strategic plan. At the same time, the CINECA consortium, a major technology partner for Italian Universities, developed a digital certification management system called Open Digital Badge Platform. As a result, collaboration with CINECA occurred and the first main outcome was the Open Digital Badge project. This represents the first stage of the process aimed at developing a Digital Academic Credential system. Since it was possible to integrate it with the existing technical platform (e.g. esse3), the Open Digital Badge project provided the inclusion of the "Diploma Supplement" in the formal certification process.

Other universities then started similar or parallel processes e.g. the University of Padua started to collaborate with UniMiB and was involved in the network of Italian universities and now other universities are interested in the project.

Blockcerts and BESTR is currently adopted by UniMiB. The University of Trento is currently evaluating the adoption of Blockcerts and BESTR for certifying their degree titles. It should be noted that BESTR is one of the most common DACS solutions available among Italian Univeristies. 


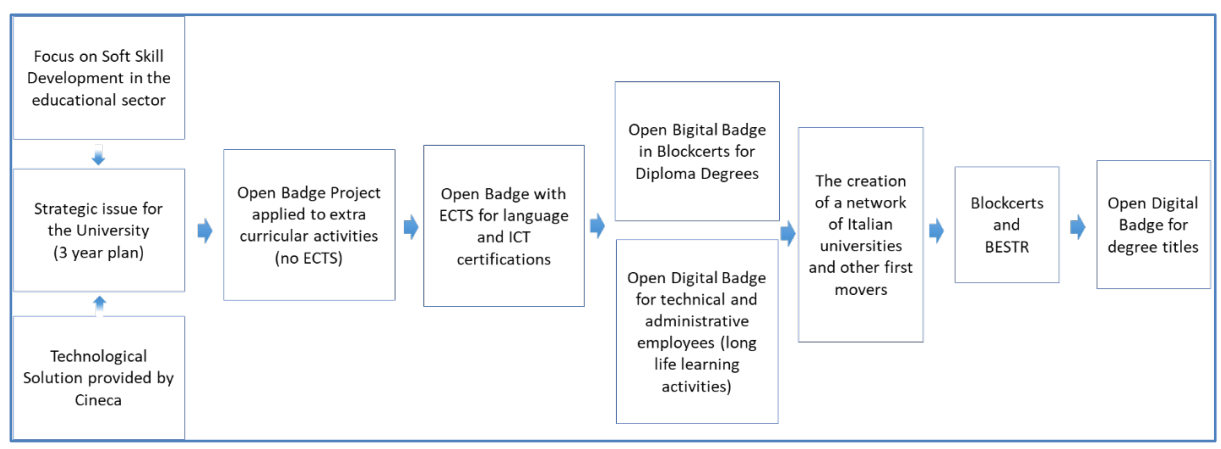

Fig. 1. - The development of a Digital Academic Credential system

\subsection{Various type of value systems with the diffusion of the DACS}

An ecosystem has been defined as "a set of actors with varying degrees of multilateral, nongeneric complementarities that are not fully hierarchically controlled" [1: 2264). Actors are here fundamental components, therefore our analysis is primarily focused on the role that various actors have in the environment.

The Fig. 2 below compares two regimes, namely "as-is" (before the emergence of the Digital Certification Ecosystem) and "to-be" (after the emergence of the Digital Certification Ecosystem).

In the situation "as-is", universities have a key role in supporting career advice and selecting students, proposing education services such as bachelor, master and doctorate programs, developing training and internship services, providing diplomas and other certificates, guaranteeing the validity of these documents and the correctness in which they are obtained, etc.. The quality is guaranteed by certification authorities at both national and international levels, which evaluate universities and other equated educational bodies, and certify their educational and training programs. During a selection process, companies usually interact directly with the candidate who declares information about diplomas and other certificates she/he obtained. In some cases, companies interact with the universities (for example when an internship is offered and/or further information is required). In northern countries in particular, companies directly contact universities to verify the certification documents provided by the candidates. With the advent and the diffusion of Digital Academic Credential System, each candidate/student/individual can create and manage a personal wallet with her/his digital badges, certificates, and diplomas.

This new scenario ("to-be" situation depicted in Fig. 2) gives rise to a complex ecosystem in which various actors interact as follows:

1. Universities certify degree and other competences through open digital badges. They may take advantage of blockchain and blockcert to guarantee the immutability and correctness of data. 
2. Other institutions may use badges or other similar mechanisms to certify courses and other competences acquired during continuance training in the professional life of employees.

3. Individuals can manage their badges, diploma and other certificates in one unique system (their digital wallet).

4. Individuals can also share this information with third parties without contacting each issuer but just showing the badges (or few of them) of their wallet.

5. Digital wallets can be consulted directly by companies without contacting the issuers.

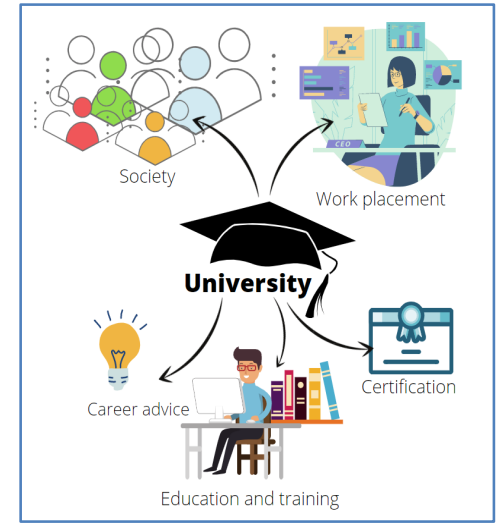

as-is

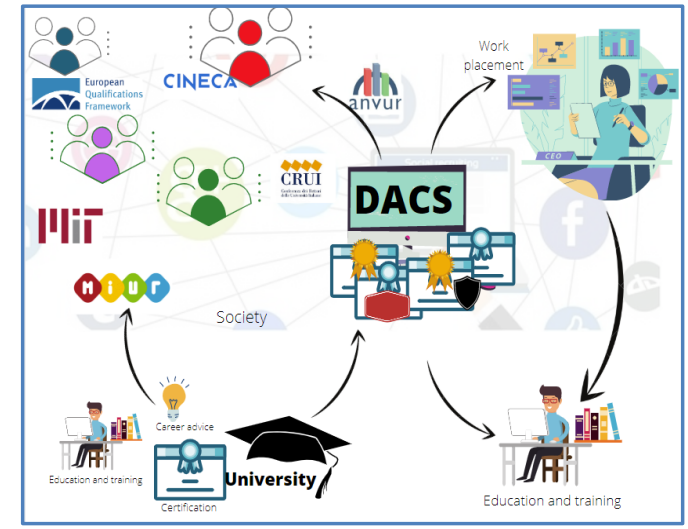

to-be

Fig. 2. The educational ecosystem. Comparison among the "as-is" and the "to-be"

In this case, universities tend to lose their previous central role in competence certification. Consequently, some resistances might occur. Indeed, in the focus group, some actors expressed a resistance. Two main reasons for this resistance can be identified. First, they do not foresee the potential cost reduction in administrative procedures (physical system). Second, they want to retain complete control, according to the traditional role of the university as the sole recognized authority of certification (structure). Contrarily, an interviewee promoting the Digital Academic Credentials considers the system as very valuable, and explained that a decentralized system of certification would radically change the role played by universities and other organizations in the educational market (structure).

Divergent opinions among informants emerged with regard to the lack of regulations. The interviewees have different expertise and attitudes toward technology and the foreseen certification systems. Overall, the interviews confirmed that this new scenario will generate a radical change and various actors and consortiums are attempting to understand it and influence it.

Diverse forces, dynamics and pressures will influence actors and institutions to adopt or to resist the development of the Digital Academic Certification System (DACS). In the light of the normative isomorphism view [19; 20], a constellation of various actors 
are investing extensive resources with the aim of introducing a new model of competence certification based on blockchain technologies. In an interview, clearly emerged that Europass aims to assist citizens, employers, and education and training authorities to define, certify and effectively communicate the content of curricula (according to national and international standards). MIT coordinates a group of leading universities and founded the Digital Academic Credential consortium aimed at designing an infrastructure for digital academic credentials and a set of international standards. This initiative wants to avoid a "lock-in" effect which could be imposed by private leading companies. In Italy, the network of the first-movers is leading the development of specific standards which the main regulatory national institutions (i.e. CRUI, ANVUR and MIUR) can be recommended as a good practice for the Italian university system. Moreover, a further normative effect could emerge from other European universities (especially in Scandinavia) that act as a valuable benchmark. Moreover other centres engaged in the field of information and consulting services relating to the procedures for the recognition of academic qualifications (such as the Italian CIMEA) are proposing blockchain based solutions open to 1) the holder of the qualification creating a personal wallet, 2) the higher education institutions which can take advantage of the ecosystem in all the phases of a study path, and 3) companies that can verify the validity of titles and qualifications.

In the light of coercive isomorphism, European Commission has put forward recommendations which underline the alignment of national certification with:

- The European Qualifications Framework, which acts as a translation device to make national qualifications more readable across Europe, promoting workers' and learners' mobility between countries and facilitating their lifelong learning;

- European Skills, Competences, Qualifications and Occupations (ESCO), a multilingual classification of skills, competences, qualifications and occupations;

- The electronic IDentification, Authentication and trust Services (eIDAS), the unique identifier that enables a verified, legally recognised digital signature.

- Other institutions, such as EFMD Programme Accreditation System, CIMEA the Italian Information Center on Mobility and Academic Equivalences, and other leading international institutions, push for the introduction of an improvement in the teaching programmes. University of Trento for instance is involved in both programs and employees involved in the field consider blockchain as a valid solution to support the holder of the qualification, higher education institutions, and certifying organizations.

In terms of mimetic isomorphism, universities may mimic and other organizations adopting the Digital Academic Certification system. Although resistance to change can be a crucial obstacle, the successful examples of other universities, perhaps those using the same information system (esse3), makes emulating others easier.

Again, about the mimetic isomorphism, it is important to underline the opinion of the head of one of the International Mobility office of the University of Trento. She has expressed the need for online certification especially abroad and especially for whom have multiple nationalities and will work in Europe or outside of Italy. This type of online verification of the correctness of diplomas becomes essential in an international 
context. In one interview, it emerged that there is a resistance to the adoption of blockchain solutions since the flow of information is not clear, the preservation of data, security, and privacy are not guaranteed and tested according to traditional standards.

\subsection{Production and consumption complementarities: value generation and benefits of the Digital Certification Ecosystems}

As explained in Jacobides et al. [1], ecosystems are based on the involvement of actors that:

- have significant interdependencies,

- provide complementary innovations, products, or services,

- belong to different industries and

- need not be bound by contractual arrangements.

These interconnections are enabled by modularity, which takes advantage of standardisation and networked externalities for digital platforms. Since the ecosystem is not hierarchically organized, the outcomes can be affected by the action of a coalition of power and first movers. In this case study, Miur, Cineca, ESCO, and other technological providers are introducing processes and rules with the attempt to coordinate actors and services provided in the ecosystem.

In this case study the presence of Digital Badges and the development of Blockchain based platform (DACS) may provide the technological modularity required to allow interdependent components of a system (the universities, companies, individuals) to share the same information with limited coordination costs.

The DACS platform coordinates a two-sided market with demand (consumption) and supply (production) sides. In the production side, issuers (e.g. the universities) provide digital badges; in the consumption side, owners, companies or third parties may take advantage of the information certified and available online. In the consumption side, various actors including EFMD, CIMEA, MIT and other partners may take advantage of these ecosystems providing new services on the DACS platform. Leading organizations can promote a broader adoption, eventually sustained also by coercive and top-down rules introduced by regulators. The interviews confirmed that a key issue related to the development of such ecosystems is the value perceived by the involved actors. Universities represent an extremely good setting to observe, calculate and unlock the value that a DACS can generate. Not only DACS is crucial in terms of competitive advantage in a task environment that places each university in an international and "stackable" market. It also provides key opportunities in terms of brand reputation, transforming into institutional value the direct and indirect endorsements. Moreover, important economic savings are introduced by the adoption of a DACS since it facilitates the digitalization and simplification of administrative processes. From the employer's point of view, a Digital Academic Credential System can dramatically reduce the amount of risk taken during recruitment processes (it is possible to verify that the certificates provided by the candidate are not counterfeit), and also increase the efficacy and efficiency of the CV screening. From a student's point of view, a DACS where all 
her/his academic achievement are stored and shared in a standardized and secure manner would clearly simplify the bureaucratic process of retriving and sending every time these pieces of information to the organization requesting them.

In our specific case study, DACS platforms - such as BESTR and Badgewallet would not exist if universities will not issue digital badges. This interdependence is fundamental to guarantee the advancement of the whole ecosystem.

The availability of digital and certified certificates enables organizations to accede in an immediate way to the validated certificates and diploma, reducing costs of $\mathrm{cv}$ screening. In the production side, the administrative cost to manage certificates will decrease because of the automation of the certification sharing process. An added value can be provided because the certification is also verified via blockchain mechanisms.

As an interviewee declared, "[DACS] and blockchain solve the problem of intermediation, fairness, integrity, etc.". He also stated: "Blockcert wallets have become a worldwide reference (MIT and 10 other universities are using them). The idea is to start with Blockerts and boost the standard by introducing the concept of consortium which has the aim of building a certification system fully controllable by the users, which can be verified digitally and securely in a distributed register.".

Finally, modularity and scalability can be boosted by the fact that in BESTR platform (the Italian platform supported by CINECA) the technology adopted is open. An interviewee states that "[...] we consider blockcert because it is an open specification to represent certificates, it can be used on blockchain (ethereum) open and private for writing or reading ... very flexible"

In Italy, the critical mass can be achieved quicker than in other countries because various services are centralised at national level. For instance, the national register of students (ANS) registers and monitors 1.5 million student careers, thus each student is uniquely identified, and the related information can be easily created and updated.

The preliminary evidence produced until now shows that significant complementarities are arising both at the production and at the consumption side. The detailed analysis of their different degrees and interconnection, according to the approach proposed in [1], is a viable and promising way to fully reveal the value generation mechanisms and drivers in the digital certification ecosystems under observation. Such developments are devoted to research in progress.

\section{Conclusions}

This paper has analysed the introduction of the Digital Academic Credential System in the university sector.

The adopted perspective enabled the authors to identify the most critical threads in the progress of the Digital Academic Credential system in all its phases of development (Figure 1). In particular, three main findings arose from the study, related to actors, ecosystem and complementarities. First (actors), we reconstructed the actions carried by some first-mover playing an important role in the Italian Digital Certification Ecosystem. Second (ecosystem), we shed light on whether and to what extent relationships among actors may change with the introduction and the adoption of Digital Academic 
Credential Systems (DACSs) based on blockchain. Third (complementarities), we identified and debated the digital ecosystem complementarities and their link to the value generation and benefits potential embedded in the DACS.

This exploratory study provides two main contributions. From the theoretical point of view, it produces a first empirical evidence to the new Ecosystem Theory proposed by Jacobides at al. [1]. It suggests that it would be valuable to carry out a deeper analysis, in particular, of the complementarities and modularities associated with the DACSs. From the managerial perspective, this study contributes to better identify and debate conditions and success factors unlocking value generation and benefits embedded in Digital Certification Ecosystems, as well as aspects that have to be further developed (e.g. privacy, legal aspects connected to the ownership of the data).

Acknowledgments. This research was supported by the Italian Ministry of Education (MIUR): “Dipartimenti di Eccellenza" Program (2018-2022) - Department of Economics and Business - University of Sassari

\section{References}

1. Jacobides, M.G., Cennamo, C., Gawer, A., 2018. Towards a theory of ecosystems. Strat Mgmt J 39, 2255-2276. https://doi.org/10.1002/smj.2904.

2. Bolici, Francesco (2019), "Blockchain as Innovation Model: a Coordination Framework for Inter-organizational Interactions", WOA2019.

3. Schoenberger, E., 1988. From Fordism to Flexible Accumulation: Technology, Competitive Strategies, and International Location. Environ Plan D 6, 245-262. https://doi.org/10.1068/d060245

4. Wenger, E., 1999. Communities of practice: Learning, meaning, and identity. Cambridge university press.

5. Nachira, F., Dini, P., Nicolai, A., 2007. A network of digital business ecosystems for Europe: roots, processes and perspectives. European Commission, Bruxelles, Introductory Paper 106.

6. Senyo, P.K., Liu, K., Effah, J., 2019. Digital business ecosystem: Literature review and a framework for future research. International Journal of Information Management 47, 52-64. https://doi.org/10.1016/j.ijinfomgt.2019.01.002

7. Cicero, S., 2018. Exploring Ecosystems: The Patterns of Platformization [WWW Document]. Medium. URL https://stories.platformdesigntoolkit.com/exploring-ecosystems-thepatterns-of-platformization-6dd0eb6f95f3 (accessed 11.4.19).

8. Suominen, A., Seppänen, M., Dedehayir, O., 2019. A bibliometric review on innovation systems and ecosystems: a research agenda. Euro Jrnl of Inn Mnagmnt 22, 335-360. https://doi.org/10.1108/EJIM-12-2017-0188

9. Ceccagnoli, M., Forman, C., Huang, P., Wu, D.J., 2011. Co-creation of value in a platform ecosystem: The case of enterprise software. MIS Quarterly, Forthcoming.

10. Wareham, J., Fox, P.B., Cano Giner, J.L., 2014. Technology ecosystem governance. Organization Science 25, 1195-1215.

11. Vargo, S.L., Wieland, H., Akaka, M.A., 2015. Innovation through institutionalization: A service ecosystems perspective. Industrial Marketing Management 44, 63-72. https://doi.org/10.1016/j.indmarman.2014.10.008 
12. Pera, R., Occhiocupo, N., Clarke, J., 2016. Motives and resources for value co-creation in a multi-stakeholder ecosystem: A managerial perspective. Journal of Business Research 69 , 4033-4041. https://doi.org/10.1016/j.jbusres.2016.03.047.

13. Beirão, G., Patrício, L., Fisk, R.P., 2017. Value cocreation in service ecosystems: Investigating health care at the micro, meso, and macro levels. Journal of Service Management 28, 227-249.

14. Cennamo, C., Santaló, J., 2019. Generativity Tension and Value Creation in Platform Ecosystems. Organization Science 30, 617-641. https://doi.org/10.1287/orsc.2018.1270.

15. de Reuver, M., Sørensen, C., Basole, R.C., 2018. The Digital Platform: A Research Agenda. Journal of Information Technology 33, 124-135. https://doi.org/10.1057/s41265-016-00333.

16. Gupta, R., Mejia, C., Kajikawa, Y., 2019. Business, innovation and digital ecosystems landscape survey and knowledge cross sharing. Technological Forecasting and Social Change 147, 100-109. https://doi.org/10.1016/j.techfore.2019.07.004.

17. Campbell, J., 2002. A critical appraisal of participatory methods in development research. International Journal of Social Research Methodology 5, 19-29.

18. Pain, R., Francis, P., 2003. Reflections on participatory research. Area 35, 46-54.

19. Meyer, J.W., Rowan, B., 1977. Institutionalized Organizations: Formal Structure as Myth and Ceremony. American Journal of Sociology 83, 340-363. https://doi.org/10.1086/226550.

20. Hinings, B., Gegenhuber, T., Greenwood, R., 2018. Digital innovation and transformation: An institutional perspective. Information and Organization 28, 52-61. https://doi.org/10.1016/j.infoandorg.2018.02.004. 